\title{
Evaluating the Driving Ability in Patients with Parkinson's Disease Using a Driving Simulator
}

\author{
Win Thiri Kyaw ${ }^{1}$, Noriko Nishikawa ${ }^{1}$, Takashi Moritoyo ${ }^{2}$, \\ Tomoaki Tsujii ${ }^{1}$, Hirotaka Iwaki ${ }^{1}$ and Masahiro Nomoto ${ }^{1}$
}

\begin{abstract}
Objective For patients with Parkinson's disease (PD), driving is challenging due to an impaired motor function and decreased attention capabilities. This study assessed the driving capacity in PD patients by comparing neurological signs.

Methods The driving ability of PD patients was evaluated using a driving simulator (Safety Master NT932) that tested the reaction time in response to traffic signals and steering wheel errors. We studied the correlations between the total Unified Parkinson's Disease Rating Scale (UPDRS) score, the UPDRS part III score, the subscores of the UPDRS part III score, age, PD disease duration, braking reaction time, steering wheel errors and total scores for driving safety test results. 'On' state regular PD licensed drivers $(\mathrm{n}=42$; mean age: 63 years) in Hoehn and Yahr stages II-III participated after their cognitive status was confirmed using mini-mental state examinations.

Results The UPDRS scores, the UPDRS part III scores and the postural instability subscores exhibited significant $(p<0.05)$ correlations with the number of steering wheel errors but not with the braking reaction time or the total safety scores of the test results.

Conclusion The UPDRS is an established evaluation method used to estimate PD signs, although it is not sufficient alone for deciding whether PD patients should be allowed to drive. Our findings suggest that determining the driving ability using a driving simulator might be a useful adjunct to UPDRS scores in the assessment of PD patients who are active drivers. Estimating the driving ability requires complex measurements, including motor performance with perception of stimuli and attention.
\end{abstract}

Key words: Parkinson's disease, driving simulator, UPDRS

(Intern Med 52: 871-876, 2013)

(DOI: 10.2169/internalmedicine.52.9292)

\section{Introduction}

Parkinson's disease (PD) is a progressive neurodegenerative disease that limits movement and sometimes affects the cognitive function. For patients with PD, driving is challenging due to impairment of the motor and non-motor functions. However, there is no universally accepted gold standard for assessing a patient's driving ability. Assessment using a combination of a driving simulator and clinical evaluations can be effective in determining driving safety, although inconsistent findings have been reported in some studies with respect to neurological PD testing and driving performance. A study of 154 PD patients found that driving ability is related to the Hoehn and Yahr scale (1), while no significant correlations were found with an on-road driving test (2).

In the present study, we investigated whether Unified Parkinson's Disease Rating Scale (UPDRS) scores or the PD disease duration can be used to evaluate the driving ability of PD patients by using a driving simulator as the standard for assessing driving ability.

${ }^{1}$ Department of Neurology and Clinical Pharmacology, Ehime University Graduate School of Medicine, Japan and ${ }^{2}$ Phase I Unit, Clinical Trial Support Center, University of Tokyo Hospital, Japan

Received for publication November 7, 2012; Accepted for publication January 7, 2013

Correspondence to Dr. Masahiro Nomoto, nomoto@m.ehime-u.ac.jp 


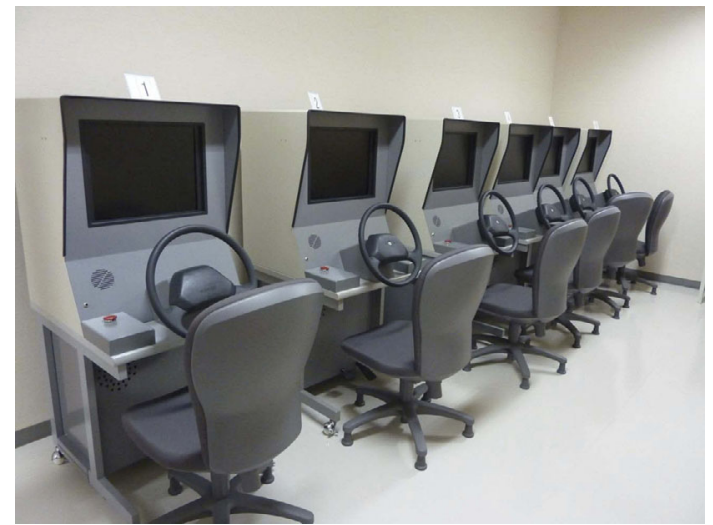

Figure 1. Driving simulator (Safety Master-NT 932).

\section{Materials and Methods}

The study was approved by the Ethical Committee of Ehime University Hospital. Written informed consent was obtained from the patients prior to participation in the study, which was performed in accordance with the principles of the Declaration of Helsinki.

PD patients in Hoehn and Yahr stages II or III were recruited for this study. The subjects were regular licensed drivers who participated in the study after they were confirmed to have a normal cognitive status on a mini-mental state examination (MMSE $\geq 27$ ).

The patients underwent a driving test using a driving simulator (Safety Master NT-932; Niigata Tsushinki Co., Ltd., Niigata, Japan) (Fig. 1) that has been officially adopted at drivers' license centers in Japan. The patients were evaluated for motor ability and perception using simple braking reaction time in response to red traffic signals, braking reaction time in response to variable signals, simple steering wheel technique and complicated steering wheel technique with braking in response to variable signals. The patients were required to handle a steering wheel to avoid colliding with a wall and to respond to changes in the colors of signals on the display by pressing the accelerator or brake pedal as necessary. In the test of simple braking reaction time in response to red traffic signals, the screen of the driving simulator displayed either a black or red traffic light. In the test of braking reaction time in response to variable signals, the patients were required to respond to four traffic lights: red, yellow, blue and black. They were required to press the accelerator in response to black or blue light, release the accelerator in response to yellow light and release the accelerator and press the braking pedal in response to a red traffic light. In the simple steering wheel test, the screen displayed the walls on the left and right sides of the road. The patients were required to avoid colliding with the walls on both sides of the road. In the complicated steering wheel technique with braking in response to variable signals test, the screen of the driving simulator displayed walls on both sides of the road and red, yellow or blue traffic lights on either side of the road. The patients were required to respond to changes in the variable signals by pressing or releasing the pedals while handling the steering wheel to prevent a collision with the walls. The total duration of the test lasted approximately 20 minutes, and the total scores of the safety driving test were rated on a 5-point scale $(1=$ very poor and unsafe, $2=$ moderately poor and unsafe, $3=$ marginal, $4=$ moderately safe and $5=$ a safe driving status).

The PD disease severity was assessed according to the UPDRS score. We evaluated the correlations between the total UPDRS score, the UPDRS part III score, the postural instability and rigidity subscores of the UPDRS part III, the PD disease duration, age, braking reaction time, the number of errors in steering and the total scores of the safety driving test results. Pearson's correlation was applied to evaluate the level of significance, with $\mathrm{p}$ values of $<0.05$ considered to be statistically significant.

\section{Results}

A total of $42 \mathrm{PD}$ patients (31 men, 11 women) with a mean $( \pm \mathrm{SD})$ age of $63 \pm 12$ years were recruited. As required by the inclusion criteria, all subjects had Hoehn and Yahr staging of II or III with a confirmed cognitive function MMSE score of $\geq 27$.

Both the UPDRS and UPDRS part III scores exhibited a tendency toward a correlation with the simple steering wheel (Fig. 2) and complicated steering wheel with braking in response to variable signals (Fig. 3) errors. The number of errors increased in association with higher UPDRS scores, although there was a significant amount of individual variability in the number of steering errors. The duration of PD was also found to be significantly correlated with the UPDRS and UPDRS part III scores (Fig. 4). However, there were no statistically significant correlations between the UPDRS and UPDRS part III scores and braking reaction time (Figs. 5, 6), the total safety score of the test results (Fig. 7) or patient age (Fig. 8). The postural instability subscore of the UPDRS part III exhibited a significant correlation with errors in the simple steering wheel test (Fig. 9A) and errors in the steering wheel with braking in response to variable signals test (Fig. 9B). However, the rigidity subscores of the UPDRS part III exhibited no correlations with any scores of the driving test (data not shown). The total number of steering wheel errors for the entire cohort exhibited a tendency to increase in association with increasing age and was similar to that observed in the healthy control subjects (Fig. 9).

\section{Discussion}

Medical practitioners are required to determine whether or not PD drivers should be allowed to continue to drive a vehicle. A prospective cohort study reported that the baseline factors for discontinuing driving in PD patients are crash history, an advanced age, low driving exposure, impairments in visual perception and cognitive abilities, parkinsonism 

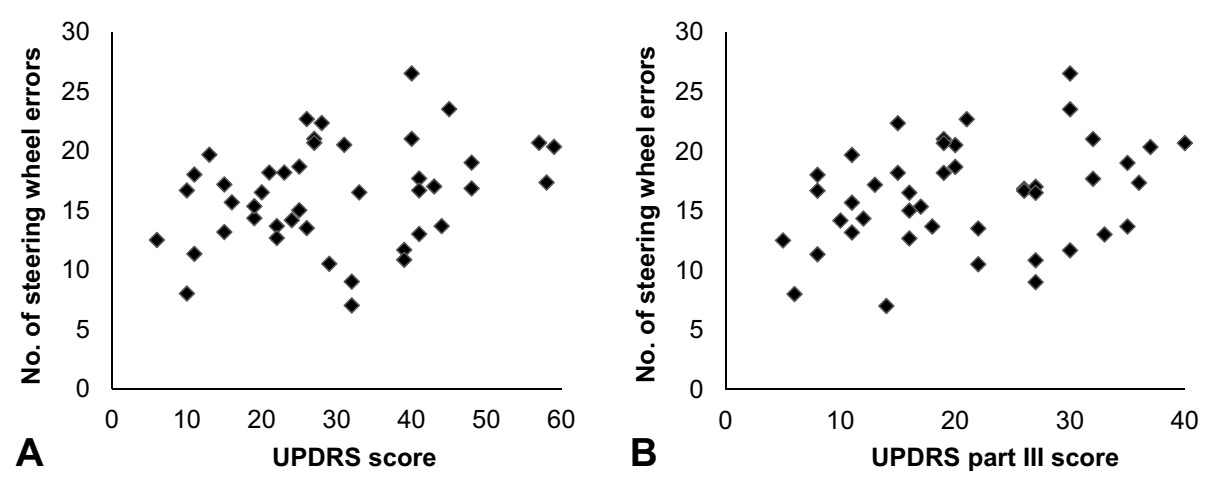

Figure 2. Correlation between the UPDRS scores and the simple steering wheel test. The number of simple steering wheel errors exhibited a tendency to increase $(\mathbf{p}<0.05)$ in association with increasing UPRDS (A) and UPDRS part III (B) scores.
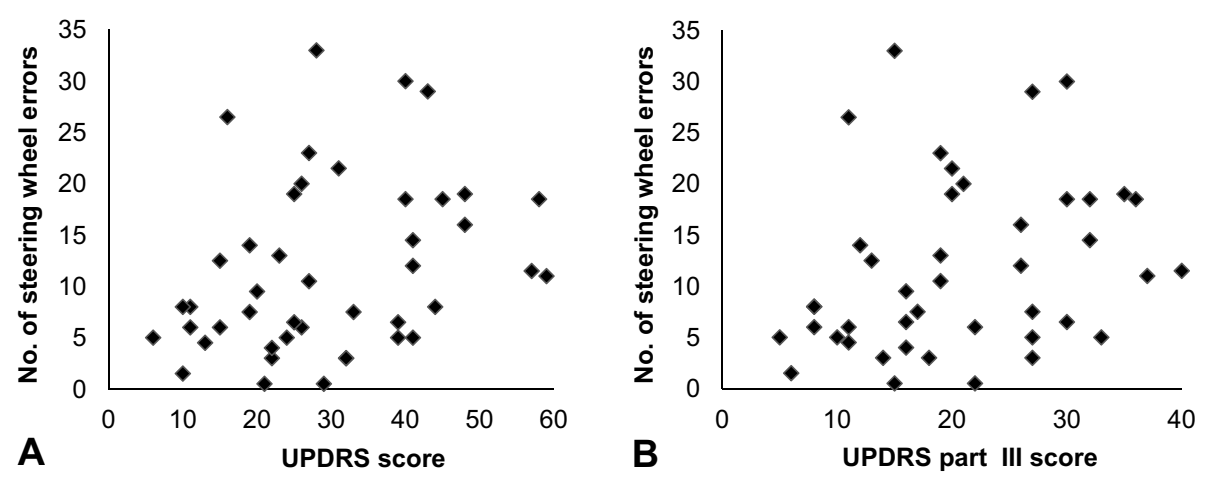

Figure 3. Correlation between the UPDRS scores and the complicated steering wheel under variable signals test. The number of steering wheel errors in response to variable signals exhibited a tendency to increase $(\mathbf{p}<\mathbf{0 . 0 5})$ in association with increasing UPDRS (A) and UPDRS part III (B) scores.
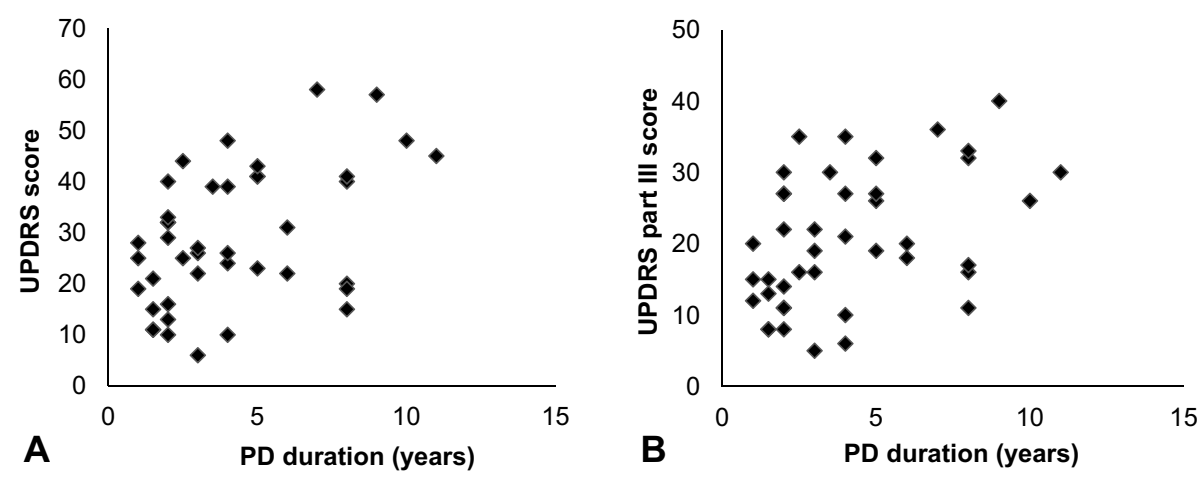

Figure 4. Correlation between the UPDRS scores and the duration of PD disease. The UPDRS (A) and UPDRS part III (B) scores significantly $(\mathbf{p}<0.05)$ increased in association with longer durations of PD disease.

and higher error counts on road tests (3). More collisions occur in patients with motor impairment and lower MMSE scores (4). PD drivers commit more safety errors on the road compared to age-matched healthy controls (5-7). Studies using driving simulators have also shown that PD patients exhibit delayed deceleration, decreased steering accuracy, slower reaction times and an increased chance of failure to respond to red traffic lights $(8,9)$. The most common factors for declining safety in PD patients are cognitive de- cline and visual processing impairment, followed by motor dysfunction and excessive daytime sleepiness (9). The driving performance of PD patients is also predicted by disease duration, visual-spatial processing and the general cognitive and motor functions (10). Audio-verbal distracted driving performance is also worse in PD patients than in controls, with declining visual sensory abilities and cognition and motor functions as causes (11). On-road and simulated driving assessments have been conducted to evaluate the driving 

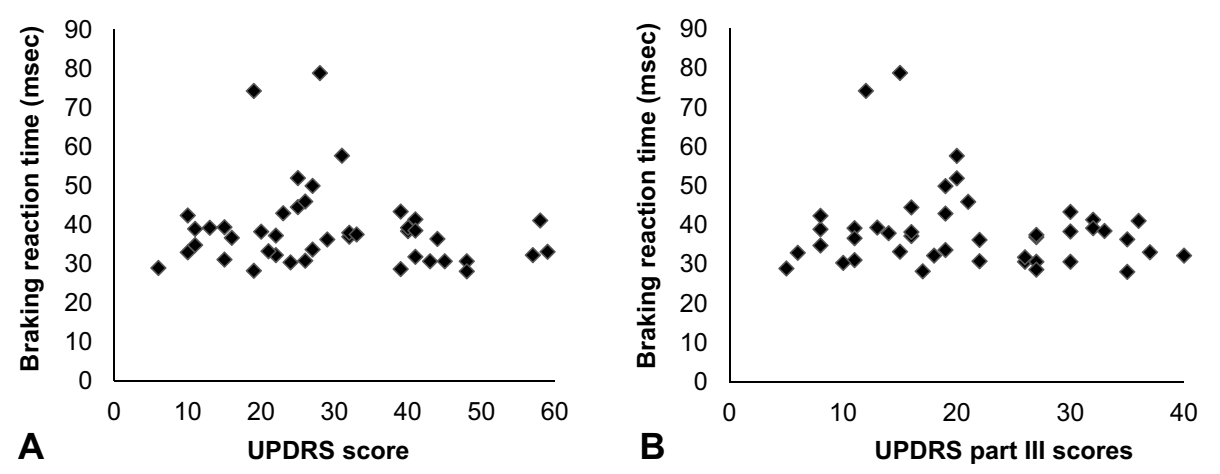

Figure 5. Correlation between the UPDRS scores and the simple braking reaction time. The simple braking reaction time in response to red traffic light signals exhibited no significant correlations with the UPDRS (A) or UPDRS part III (B) scores.
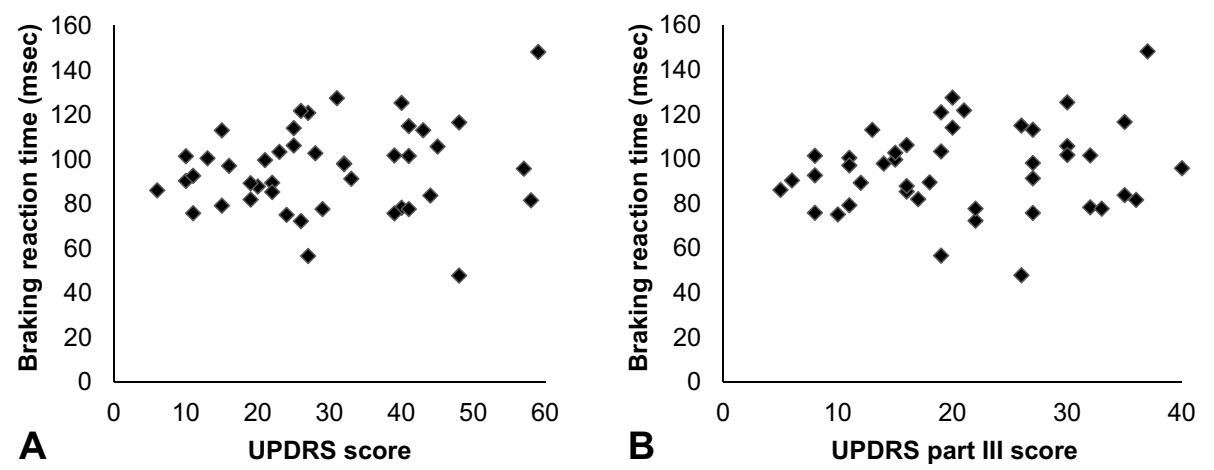

Figure 6. Correlation between the UPDRS scores and the braking reaction time under variable signals. The braking reaction time in response to variable signals exhibited no significant correlations with the UPDRS (A) or UPDRS part III (B) scores.
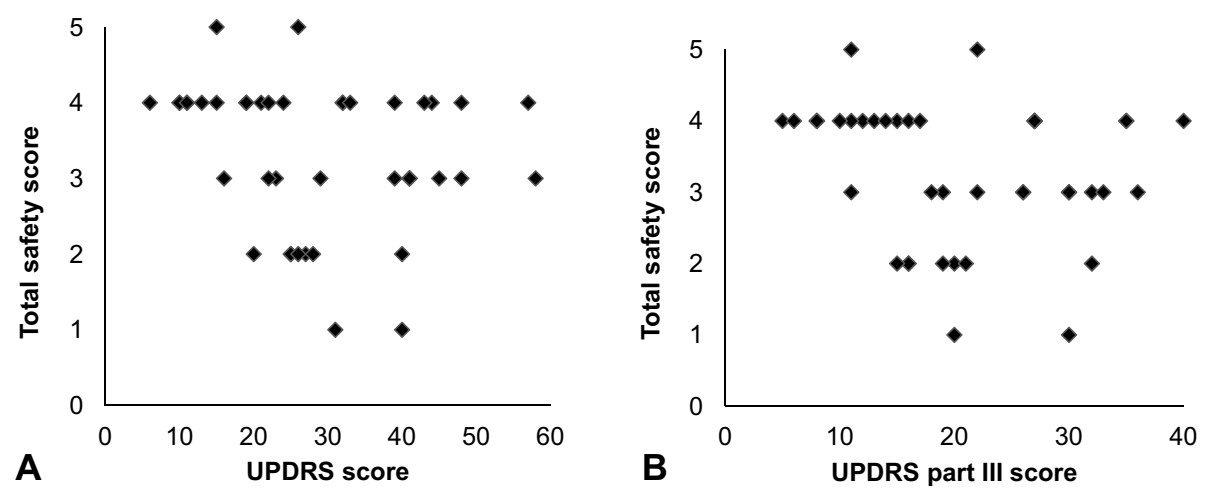

Figure 7. Correlation between the UPDRS scores and the total driving safety test scores. The total driving safety test scores exhibited no significant correlations with the UPDRS scores.

safety of PD patients with mild to moderate disease severity under medication. Webster's rating scale evaluating the 10 key disease criteria of PD, including bradykinesia, rigidity, posture, upper extremity swing, gait, tremor, facies, seborrhea, speech and self-care (12), has also been applied in some studies. In a simulated driving assessment study using Webster's rating scale as the measurement of motor severity in PD patients (8), the Webster's rating scale score was found to be significantly correlated with the driving reaction time, steering accuracy and simple reaction time. However, another study using a similar study design reported that Webster's rating scale is not a predictor of driving assessment in PD patients (13).

In our study, we found that steering accuracy was increasingly impaired in relation to disease severity. Both steering wheel performance and PD disease duration were found to be significantly related to the UPDRS score during the evaluation of driving ability among PD patients using a 

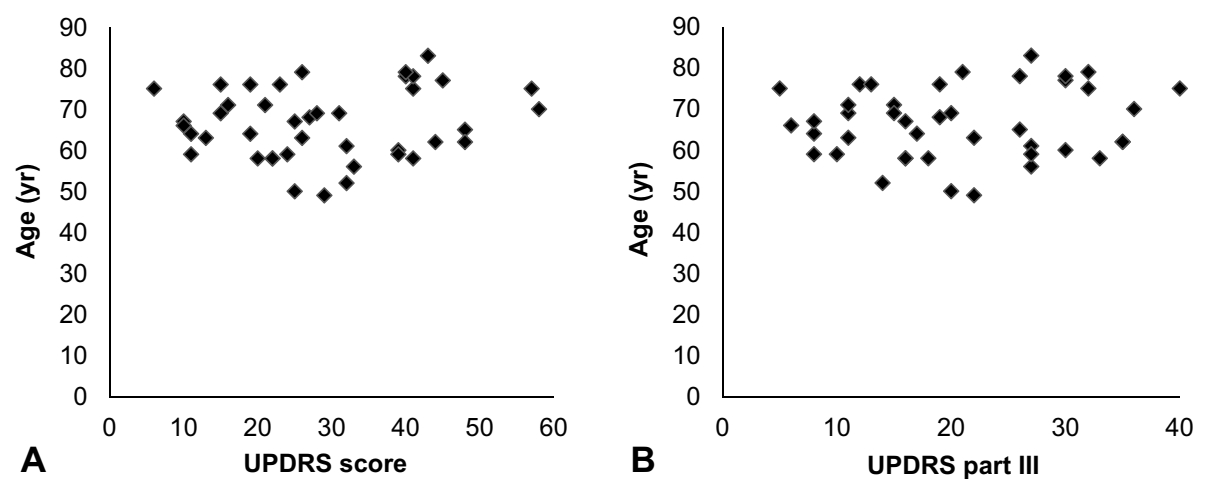

Figure 8. Correlation between the UPDRS scores and patient age. Patient age exhibited no correlations with the UPDRS (A) or UPDRS part III (B) scores.
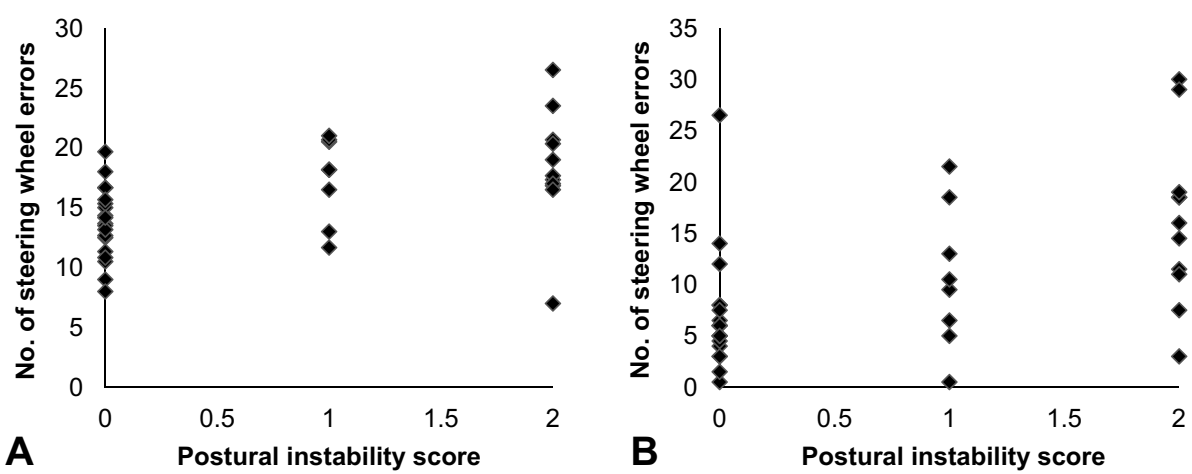

Figure 9. Correlation between the postural stability subscores of the UPDRS part III and the steering wheel test scores. The number of simple steering wheel errors $(A)$ and the steering wheel response to variable signals scores $(B)$ significantly $(\mathrm{p}<0.01$ and $\mathrm{p}<0.001$, respectively) increased in association with increasing postural instability scores.

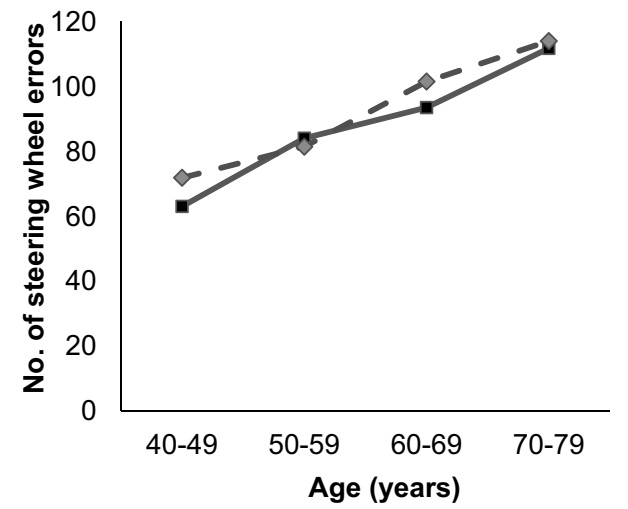

Figure 10. Correlation between the simple steering wheel test and age among the PD patients (solid line) and agematched control subjects (dashed line). The total number of steering wheel errors in the PD cohort increased in accordance with higher ages. This increase was similar to that observed in the matched control subjects. The values of the normal control subjects were obtained from an in-house document from the driving test simulator manufacturer (Niigata Tsushinki Co., Ltd., Niigata, Japan).

driving simulator. The patients with impaired postural stabil- ity made more errors in handling the steering wheel. In contrast, the UPDRS score exhibited no significant correlations with the braking reaction time or the driving safety test results, consistent with the findings of a previous report of onroad assessment (14). The braking action response to traffic signals depends on the perception of stimuli that cannot be assessed by the UPDRS. The UPDRS score alone, therefore, is not adequate to determine whether PD patients should be allowed to drive. Our findings suggest that evaluating the driving ability using a driving simulator might be a useful adjunct to UPDRS scores in the assessment of PD patients who are active drivers.

The authors state that they have no Conflict of Interest (COI).

Grant support: The work was supported by Grants-in-Aid from the Research Committee for Parkinson's Disease, the Ministry of Health, Labour and Welfare of Japan and SRFJ and a Grant from Ehime University.

\section{References}

1. Singh R, Pentland B, Hunter J, Provan F. Parkinson's disease and driving ability. J Neurol Neurosurg Psychiatry 78: 363-366, 2007. 
2. Heikkilä VM, Turkka J, Korpelainen J, Kallanranta T, Summala H. Decreased driving ability in people with Parkinson's disease. J Neurol Neurosurg Psychiatry 64: 325-330, 1998.

3. Uc EY, Rizzo M, Johnson AM, et al. Real-life driving outcomes in Parkinson disease. Neurology 76: 1894-1902, 2011.

4. Uc EY, Rizzo M. Driving and neurodegenerative disease. Curr Neurol Neurosci Rep 8: 377-383, 2008.

5. Wood JM, Worringham C, Kerr G, Mallon K, Silburn P. Quantitative assessment of driving performance in Parkinson's disease. J Neurol Neurosurg Psychiatry 76: 176-180, 2005.

6. Cordell R, Lee HC, Granger A, Vieira B, Lee AH. Driving assessment in Parkinson's disease: a novel predictor of performance? Mov Disord 23: 1217-1222, 2008.

7. Uc EY, Rizzo M, Johnson AM, et al. Road safety in drivers with Parkinson's disease. Neurology 73: 2112-2119, 2009.

8. Madeley P, Hulley JL, Wildgust H, Mindham RH. Parkinson's disease and driving ability. J Neurol Neurosurg Psychiatry 53:
580-582, 1990.

9. Stolwyk RJ, Triggs TJ, Charlton JL, Moss S, Iansek R, Bradshaw JL. Effect of a concurrent task on driving performance in people with Parkinson's disease. Mov Disord 21: 2096-2100, 2006.

10. Uitti RJ. Parkinson's disease and issues related to driving. Parkinsonism Relat Disord 15 (Suppl. 3): S122-S125, 2009.

11. Uc EY, Rizzo M, Anderson SW, Sparks JD, Rodnitzky RL, Dawson JD. Driving with distraction in Parkinson's disease. Neurology 67: 1774-1780, 2006.

12. Webster DD. Critical analysis of the disability in Parkinson's disease. New York: Mod Treat 5: 257-282, 1968.

13. Lings S, Dupont E. Driving with Parkinson's disease. A controlled laboratory investigation. Acta Neurol Scand 86: 33-39, 1992.

14. Grace J, Amick MM, D'Abreu AD, Festa EK, Heindel WC, Ott BR. Neuropsychological deficits associated with driving performance in Parkinson's and Alzheimer's disease. J Int Neuropsychol Soc 11: 766-775, 2005.

(C) 2013 The Japanese Society of Internal Medicine http://www.naika.or.jp/imonline/index.html 